\title{
Structural Analysis of Dextran-Based Hydrogels Obtained Chemoenzymatically
}

\author{
L. Ferreira, ${ }^{1}$ M. M. Figueiredo, ${ }^{2}$ M. H. Gil, ${ }^{2}$ M. A. Ramos ${ }^{3}$ \\ ${ }^{1}$ INEB-Instituto de Engenharia Biomédica, Laboratório de Biomateriais, Rua do Campo Alegre 823, 4150-180 \\ Porto, Portugal \\ ${ }^{2}$ Departamento de Engenharia Química, Universidade de Coimbra, Pinhal de Marrocos, 3030 Coimbra, Portugal \\ ${ }^{3}$ Instituto Superior de Engenharia, Inst. Politécnico de Coimbra, 3000 Coimbra, Portugal
}

Received 13 March 2005; revised 9 May 2005; accepted 2 June 2005

Published online 6 October 2005 in Wiley InterScience (www.interscience.wiley.com). DOI: 10.1002/jbm.b.30394

\begin{abstract}
This work reports the results of structural analysis in novel dextran-acrylate (dexT70-VA) hydrogels generated chemoenzymatically. Porous structure as well as hydrogel surface and interior morphologies were evaluated by mercury intrusion porosimetry (MIP), nitrogen adsorption (NA), and scanning electron microscopy (SEM) analyses, as a function of the degree of substitution (DS), and initial water content used in the preparation of the hydrogel. MIP analysis showed that the overall networks were clearly macroporous with pore sizes ranging from 0.065 to $10 \mu \mathrm{m}$. As expected, the average pore size decreased as DS increased and as initial water content decreased. Moreover, the porosity values ranged from 75 up $90 \%$, which shows that these hydrogels present an interconnected pore structure. Nitrogen adsorption analyses showed that the specific surface area of dexT70-VA hydrogels increased either by increasing the DS or by decreasing the initial water content of the hydrogel. SEM results revealed that the surface of hydrogels with lower DS presented either a porous structure or a polymeric "skin" covering the pores, whereas hydrogels with higher DS were totally porous. Furthermore, the interior morphology varied according to the DS and the initial water content of the hydrogels. Finally, the average pore size was also determined from the swelling of hydrogel using a theoretical model developed by Flory-Rehner. The comparison of the SEM and MIP results with the ones obtained by the equilibrium swelling theory of Flory-Rehner shows that this approach highly underestimates the average pore size. (C) 2005 Wiley Periodicals, Inc. J Biomed Mater Res Part B: Appl Biomater 77B: 55-64, 2006
\end{abstract}

Keywords: hydrogels; scanning electron microscopy; mercury intrusion porosimetry; gas adsorption; structural characterization; morphology

\section{INTRODUCTION}

Hydrogels are interesting systems for peptide and protein delivery because of their good tissue biocompatibility and possibilities to manipulate the permeability for solutes. ${ }^{1,2}$ In addition, hydrogels are an exciting approach to cell delivery for tissue engineering. ${ }^{3,4}$ Irrespective of their use, it is crucial to perform structural analysis to evaluate pore size and morphology, porosity, and surface area, among other parameters. These parameters are critical factors to control swelling, drug release behavior, and biological interaction inside the body. ${ }^{5-7}$ In fact, it has been reported that pore-size distribution in degrading dextran-methacrylate hydrogels is very

Correspondence to: L. Ferreira (e-mail: lino@mit.edu, 1sf@ineb.up.pt)

Contract grant sponsor: Fundação para a Ciência e a Tecnologia; contract grant number: Praxis XXI, BD/18456/98 (to L.F)

(C) 2005 Wiley Periodicals, Inc. important to modulate the release of proteins. ${ }^{5}$ In addition, it has been shown that subcutaneous implants, which had virtually no pores larger than $5 \mu \mathrm{m}$ were not integrated in the surrounding tissue, whereas the opposite was found for implants with pores larger than $10 \mu \mathrm{m} .{ }^{6,8}$ A higher degree of tissue integration enhances drug and cell delivery of implantable hydrogel devices. Furthermore, porosity, which gives an indication of the pore connectivity, is important to assess the possibility of blood vessel ingrowth necessary for cell-transplanted activity. ${ }^{6}$

Hydrogel pore structure can be assessed by mercury intrusion porosimetry (MIP), thermoporometry, and nitrogen adsorption or by visual methods like scanning electron microscopy. Thermoporometry, recently applied in the field of hydrogels, ${ }^{9}$ provides information on pore size distributions in the mesopore range (pores with diameters between 2 and 50 $\mathrm{nm}^{10}$ ) in swollen hydrogels; however, the feasibility of using this technique for macropores (pores with diameters higher 
than $50 \mathrm{~nm}^{10}$ ) assessment has not yet been reported. SEM is very often described in the literature to study morphological details of cryofixed-swollen hydrogels. ${ }^{11-14}$ Indeed, image analysis has been used to obtain information about pore size distribution, porosity, and surface area density; ${ }^{11}$ however, such information is restricted to the location where the analyses are performed. MIP is widely accepted as a standard method to measure porosity, and it also allows an estimation of pore size distribution in the macro- and mesopore ranges, while nitrogen adsorption (NA), currently used for surface area determinations, is capable of measuring pore sizes in the micro- (pores with diameters lower than $2 \mathrm{~nm}^{10}$ ) and mesopore ranges. Unfortunately, few studies have used both techniques for pore structural analysis in hydrogels. ${ }^{6,12,15}$

Recently, we have reported the preparation of dextranacrylate (dexT70-VA) hydrogels by chemoenzymatic routes and determined the average pore diameter through the equilibrium swelling theory of Flory-Rehner. ${ }^{16,17}$ This is a simple and widely used approach to estimate pore sizes, ${ }^{18-21}$ only requiring the experimental determination of polymer volume fractions, which are determined from hydrogel swelling. However, a deeper characterization of structural properties of dexT70-VA hydrogels was not performed. In the present work, the morphology, porosity, surface area, and pore size distribution of dextran-based hydrogels were evaluated by MIP, NA, and SEM analyses. Furthermore, the pore size distributions given by the different techniques were compared with the values obtained through modeling by the equilibrium swelling theory of Flory-Rehner.

\section{MATERIALS AND METHODS}

\section{Materials}

Dextran (from Leuconostoc mesenteroides, dexT70, $M_{n}=$ $39,940, M_{w}=70,000$, according to the manufacturer's specification) was obtained from Fluka Chemie AG (Buchs, Switzerland). Vinyl acrylate (VA), dimethylsulfoxide (DMSO), $\mathrm{N}, \mathrm{N}, \mathrm{N}^{\prime}, \mathrm{N}^{\prime}$-tetramethylenediamine (TEMED), and ammonium persulfate (APS) were purchased from Aldrich (Milwauke, WI). Dextran acrylates (dexT70-VA) with different degree of substitution (DS) were synthesized as described previously. ${ }^{16}$ The products were characterized by ${ }^{1} \mathrm{H}$ NMR to assess the DS. ${ }^{16}$ All other chemicals and solvents used in this work were of the highest purity commercially available.

\section{Preparation of dexT70-VA Hydrogels}

Dext70-VA hydrogels were obtained by free radical polymerization of aqueous solutions of dexT70-VA as a function of DS and monomer concentration. Dext70-VA (80 or $200 \mathrm{mg}$ ) was dissolved in $0.9 \mathrm{~mL}$ of $0.2 \mathrm{M}$ phosphate buffer, $\mathrm{pH} 8.0$, and bubbled with nitrogen for $2 \mathrm{~min}$. The polymerization reactions, performed in a cell culture plate (diameter $\cong 1.8$ $\mathrm{cm}$ ) (in case of bubbles, they were removed with a pipette tip), were initiated by adding $50 \mu \mathrm{L}$ APS $(80 \mathrm{mg} / \mathrm{mL}$ in 0.2 $M$ phosphate buffer, $\mathrm{pH} 8.0$ ) and $50 \mu \mathrm{L}$ TEMED solution
(13.6\% (v/v) in water; $\mathrm{pH}$ adjusted to 8.0 with $12 \mathrm{~N} \mathrm{HCl}$; this compound accelerates the rate formation of free radicals from APS), and allowed to proceed for $24 \mathrm{~h}$ at $25^{\circ} \mathrm{C}$. The hydrogels synthesized contained an initial water content of $92 \%(\mathrm{w} / \mathrm{w})$ and $80 \%$ (w/w), when $80 \mathrm{mg}$ and $200 \mathrm{mg}$ of dexT70-VA macromonomer were used, respectively, maintaining constant the other components (i.e., buffer, APS, and TEMED).

The hydrogels, after being removed from the plate, were immersed in ca. $50 \mathrm{~mL}$ of $0.01 M$ citrate-phosphate buffer, $\mathrm{pH} 7.0$, changing the buffer daily, at $25^{\circ} \mathrm{C}$. After 5 days, the hydrogels disks were steam-sterilized for $20 \mathrm{~min}$ at $120^{\circ} \mathrm{C}$ followed by equilibration for $24 \mathrm{~h}$ at $25^{\circ} \mathrm{C}$. At this time the swollen weight $\left(W_{s}\right)$ of hydrogels was determined. The hydrogels were then dried at room temperature, under vacuum, in the presence of phosphorous pentoxide (until constant weight was achieved), and weighed to determine the dried weight, $W_{d}$. The swelling ratio at equilibrium (SRE) was calculated according to: $\operatorname{SRE}=\left(W_{s}-W_{d}\right) / W_{d}$.

For SEM, MPI, and NA analyses, the hydrogels after being steam sterilized were flash frozen in liquid nitrogen and lyophilized on a freeze drier (FTS System, NY; temperature ca. $-89^{\circ} \mathrm{C}$ and pressure below $100 \mathrm{mTorr}$ ) for $24 \mathrm{~h}$.

\section{Structural Characterization through the Equilibrium Swelling Theory}

The molecular weight between the crosslinks $\left(\bar{M}_{c}\right)$ was calculated by the equilibrium swelling theory of Flory and Rehner ${ }^{17}$ and modified by Peppas et al. ${ }^{18}$ For the case of highly crosslinked networks, where the crosslinks were introduced in solution, the $\bar{M}_{c}$ was calculated according to Equation (1), which takes into account deviations from a Gaussian distribution of polymer chain lengths:

$$
\begin{aligned}
\frac{1}{\bar{M}_{c}}= & \frac{2}{\bar{M}_{n}} \\
& -\frac{\left(\frac{\bar{\nu}}{V_{1}}\right)\left[\ln \left(1-\nu_{2, s}\right)+\nu_{2, s}+\chi_{1}\left(\nu_{2, s}\right)^{2}\right]\left[1-\frac{1}{\chi_{c}}\left(\frac{\nu_{2, s}}{\nu_{2, r}}\right)^{2 / 3}\right]^{3}}{\nu_{2, r}\left[\left(\frac{\nu_{2, s}}{\nu_{2, r}}\right)^{1 / 3}-0.5\left(\frac{\nu_{2, s}}{\nu_{2, r}}\right)\right]\left[1+\frac{1}{\chi_{c}}\left(\frac{\nu_{2, s}}{\nu_{2, r}}\right)^{1 / 3}\right]^{2}}
\end{aligned}
$$

where $\bar{M}_{n}$ is the number average molecular weight of dextran $(39,940 \mathrm{Da}), \bar{\nu}$ is the partial specific volume of dextran $(0.62$ $\left.\mathrm{cm}^{3} / \mathrm{g}\right), V_{1}$ is the molar volume of water $\left(18 \mathrm{~cm}^{3} / \mathrm{g}\right), \chi_{1}$ is the Flory polymer-solvent interaction parameter $(0.473$ for dextran/water $), \chi_{c}$ is the number of links of the chain $\left(\chi_{c}=\right.$ $2 M_{c} M_{r}$, where $M_{r}$ is the molecular weight of the dextran repeating unit, 162.14), $\nu_{2, r}$ is the polymer fraction of the gel after gel formation and $\nu_{2, s}$ is the polymer fraction at equilibrium swelling. $\nu_{2, r}$ and $\nu_{2, s}$ were calculated from the weight of the gels before exposure to the buffer solution and after equilibrium swelling, respectively, assuming volume additivity of water and dextran. 
The average mesh size, $\xi$, was calculated from the $\bar{M}_{c}$ values through the use of Equations (2) and (3): ${ }^{19}$

$$
\begin{aligned}
& \bar{r}_{0}^{2}=C_{n} \frac{2 \overline{M_{c}}}{M_{r}} b^{2} \\
& \xi=v_{2, s}{ }^{-1 / 3}\left(\bar{r}_{0}^{2}\right)^{1 / 2}
\end{aligned}
$$

where $\bar{r}_{0}^{2}$ represents the average end-to-end subchain length (in $\AA$ ) when the gel is unswollen, $C_{n}$ is the polymer rigidity factor, assumed to be 8.9 by analogy to polar poly(vinyl alcohol), ${ }^{19} M_{r}$ is the molecular weight of the dextran repeating unit $\left(162.14 \mathrm{gmol}^{-1}\right), b$ is the characteristic bond length of the polymer backbone ( $=1.54 \AA$, corresponding to the $\mathrm{C}-\mathrm{C}$ bond length) and $v_{2, s}$ is the polymer fraction at equilibrium swelling.

\section{MIP}

MIP (Micromeritics Poresizer 9320) was used to determine bulk density, skeletal density, porosity, and pore size distribution. All the samples were degassed before analysis at a vacuum pressure below $50 \mu \mathrm{mHg}$. By knowing the volume intruded at 1-2 psia (known as bulk volume, as it includes solid sample, pores, and interstices) and the material weight, it is possible to calculate the bulk density. High pressure runs (from 25 up to 30,000 psia) were performed with an equilibration time of $20 \mathrm{~s}$ and a maximum intrusion volume of $0.0500 \mathrm{~mL} / \mathrm{g}$. At 30,000 psia the skeletal density (calculated from the skeletal volume measured at this pressure, which includes solid sample and pores diameters bellow $6 \mathrm{~nm}$ ) was determined. The porograms (intruded volume vs. pressure) obtained were converted into pore size distribution curves (cumulative and differential) according to the Washburn equation: ${ }^{22}$

$$
p d=-4 \gamma \cos \theta
$$

where $p$ is the pressure required to force mercury into a pore of entry diameter $d, \gamma$ is the mercury surface tension (485 dyn $\mathrm{cm}^{-1}$ ), and $\theta$ is the contact angle between mercury and the sample $\left(130^{\circ}\right)$. The porosity of hydrogels was calculated from Equation (5):

porosity

$$
=((\text { skeletal density-bulk density }) / \text { skeletal density }) \times 100
$$

\section{NA}

NA isotherms of hydrogels were obtained using ASAP (Accelerated Surface Area and Porosimetry) 2000 from Micromeritics. The samples were previously degassed to below 5 $\mu \mathrm{mHg}$ at room temperature and the analyses were performed at $77 \mathrm{~K}$, using liquid nitrogen. The equilibration interval was $5 \mathrm{~s}$. The surface area was calculated using the BET method. ${ }^{23}$
Pore size distribution, based on BJH calculations, ${ }^{24}$ was evaluated by the apparatus software.

\section{SEM}

Scanning electron micrographs were obtained from swollen freeze-dried gels (fractured pieces of $0.6-0.9 \mathrm{~cm}$ in length, corresponding to half-diameter of each hydrogel) mounted onto an aluminum stud, and gold coated by plasma vapor deposition. The hydrogel surface and interior were recorded by a field emission scanning electron microscope (JEOL model JSM-5310), at $15.0 \mathrm{kV}$. In both regions representative pore size distributions, at two different radial positions, were photographed.

Analyses of the digitized images were performed using Scion Image (Scion Corporation, Maryland), according to a methodology described by others. ${ }^{11,12}$ Briefly, a thresholding procedure was applied to the SEM images and the validity of the thresholding level was confirmed by comparing the image before and after the procedure. In the case of irregularities, they were corrected manually by comparison to the original photograph. After calibrating with a known scale, the pores were measured and labeled one by one. The diameter of a pore was obtained by averaging its major and minor axes.

\section{Rheological Characterization}

Rheological experiments have been carried out using the parallel plate geometry (20 mm diameter, steel) of a Haake Rheostress RS 1. Sandpaper has been glued onto each plate surface to avoid slippage of samples. Equilibrium swelling conditions have been maintained during rheological measurements by adding water at $\mathrm{pH} 7$ on the lower plate of the geometry until the entire free lateral surface of hydrogels has been completely wetted by the liquid. Gap setting optimizations have been undertaken according to the procedure described elsewhere. ${ }^{25}$ Hydrogels were subjected to stress sweep experiments (frequency of $1 \mathrm{~Hz}$ ) to optimize the applied stress used in the frequency-oscillation experiments. These last experiments were recorded over a frequency range from 0.1 to $10 \mathrm{~Hz}$.

\section{RESULTS AND DISCUSSION}

Regioselective incorporation of acrylate groups in the dextran backbone was accomplished by transesterification of dexT70 with VA catalyzed by Proleather, a Bacillus subtilis protease, dissolved in DMSO ${ }^{16}$ Dextran-acrylate (dexT70-VA) hydrogels were obtained upon free radical polymerization of aqueous solutions of dexT70-VA (Fig. 1). Because we envision to apply these hydrogels in vivo, ${ }^{26}$ they were sterilized by autoclave prior their characterization. The cryofixed swollen dextran-acrylate hydrogels were characterized by MIP, NA, and SEM to fully evaluate their pore structure. 


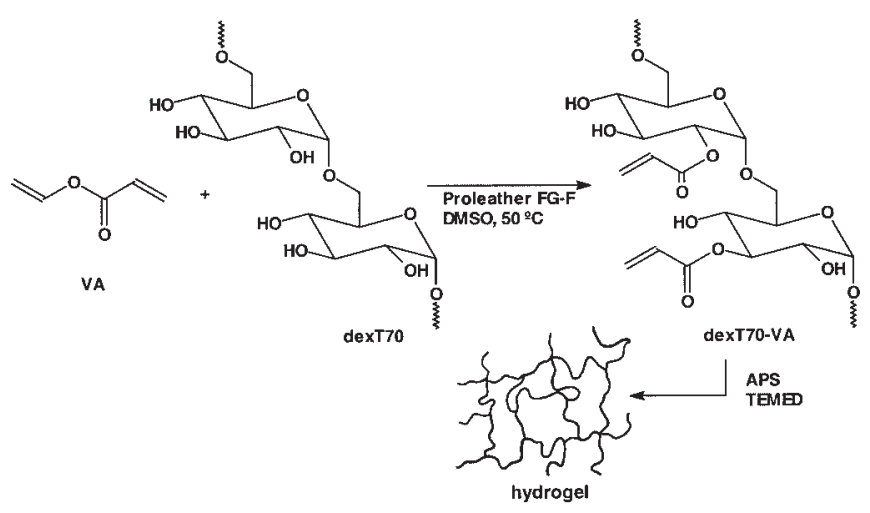

Figure 1. Schematic representation of the preparation of dexT70-VA hydrogels.

\section{Structural Analysis by MIP}

This technique measures the intruded volume of mercury in the sample at specific pressures. Afterwards, the pressure is converted into pore size according to the mathematical model of Washburn [Equation (4)], which assumes that the applied pressure is inversely proportional to the pore (cylindrical) diameter.

Figure 2 shows the intrusion curves for dexT70-VA hydrogels obtained from initial water content of $80 \%$ as a function of the degree of substitution. In general, the curves show a small intrusion of mercury (less than one-third of the total mercury volume intruded in the sample) for pressures below 30 psia, corresponding to pore diameters larger than ca. $10 \mu \mathrm{m}$ and a predominant intrusion for pressures above 200 psia, effectively corresponding to hydrogel pore diameters below $1 \mu \mathrm{m}$, as will be confirmed by nitrogen adsorption and SEM analyses (see below). As clearly shown by the high content of mercury intruded (also displayed in Table I), hydrogels with low DS, particularly those with a DS of $7.2 \%$,

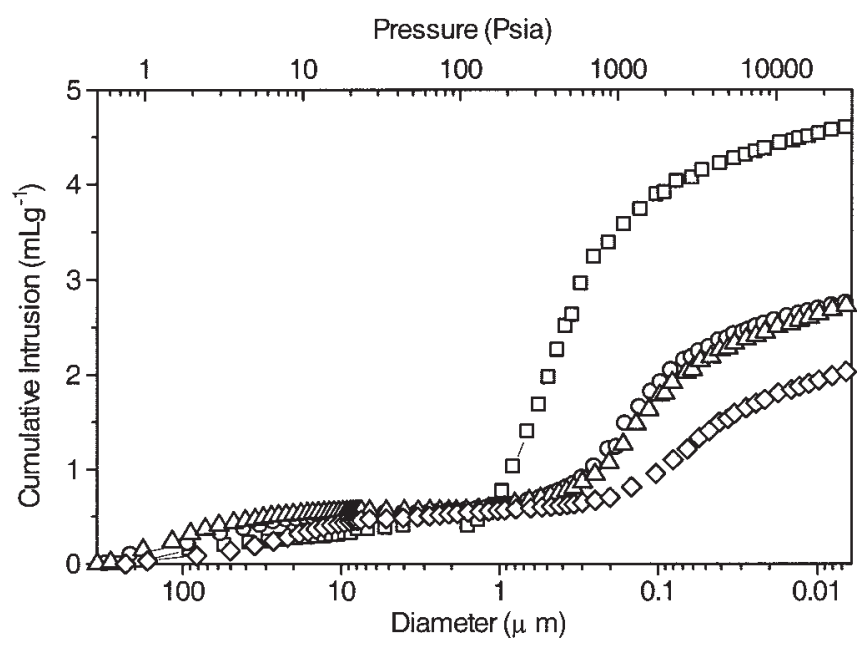

Figure 2. Mercury intrusion curves (volume vs. pressure/diameter) of dexT70-VA hydrogels with $80 \%$ initial water content and various degrees of substitution: DS $7.2 \%(\square)$, DS $12.1 \%(\bigcirc)$, DS $22.4 \%(\triangle)$, DS $31.5 \%(\diamond)$.
TABLE I. Characteristics of Swollen dexT70-VA Hydrogels with Different Initial Water Content and Degree of Substitution (DS), as Measured by MIP

\begin{tabular}{|c|c|c|c|c|}
\hline $\begin{array}{c}\text { DexT70-VA } \\
\text { hydrogel }\end{array}$ & $\begin{array}{l}\text { Volume of } \\
\text { Mercury } \\
\text { Intruded } \\
\left(\mathrm{mLg}^{-1}\right)\end{array}$ & $\begin{array}{c}\text { Pore Size } \\
\text { Range } \\
(\mu \mathrm{m})^{\mathrm{a}}\end{array}$ & $\begin{array}{c}\text { Pore } \\
\text { diameter }^{\mathrm{b}} \\
\quad(\mu \mathrm{m})\end{array}$ & $\begin{array}{c}\text { Porosity } \\
(\%)^{\mathrm{c}}\end{array}$ \\
\hline $92 \%$, DS $7.2 \%$ & 18.92 & $0.15-210$ & 10 & 88.9 \\
\hline $92 \%$, DS $12.1 \%$ & 7.85 & $0.12-4.4$ & 2.0 & 89.6 \\
\hline $92 \%$, DS $22.4 \%$ & 6.80 & $0.16-3.8$ & 0.80 & 87.0 \\
\hline $92 \%$, DS $31.5 \%$ & 5.75 & $0.26-1.75$ & 0.65 & 81.0 \\
\hline $80 \%$, DS $7.2 \%$ & 4.60 & $0.059-3.2$ & 0.50 & 87.6 \\
\hline $80 \%$, DS $12.1 \%$ & 2.76 & $0.056-0.34$ & 0.20 & 79.3 \\
\hline $80 \%$, DS $22.4 \%$ & 2.72 & $0.047-0.28$ & 0.17 & 78.1 \\
\hline $80 \%$, DS $31.5 \%$ & 2.03 & $0.035-0.16$ & 0.07 & 74.6 \\
\hline
\end{tabular}

present higher pore volumes than hydrogels with high DS. This is due to the low number of crosslink points, which makes these networks more open and thus with more empty spaces.

Figure 3 shows the pore size differential distributions for the overall dextran-based hydrogels. Taking into account the pore size range of the networks, hydrogels with low DS exhibit broader pore size distributions than networks with high DS. From Figure 3 and Table 1 it can also be concluded that the pore sizes of hydrogels (represented by the modes of the differential distribution curves, that is, the most frequent diameter) decrease as DS increases, as a result of the increased number of intermolecular crosslinks, which are favored by the higher number of acrylate groups attached to dextran. However, this effect is somehow less pronounced for higher values of DS, which might be related to the low efficiency of the crosslinking reaction in this range of DS. In fact, previously, we demonstrated by FTIR experiments ${ }^{15}$ that the polymerization of vinyl groups in dexT70-VA monomers with high DS, was not as efficient as the polymerization of monomers with low DS.

The initial water content in the polymerization reaction played also an important effect (in some cases more relevant than DS) in the pore volume and pore size distribution on dexT70-VA hydrogels. Indeed, hydrogels with higher initial water content exhibit higher pore volumes and pore sizes than hydrogels with lower initial water content (Fig. 3 and Table 1). This is due to the increase of intermolecular crosslinks and physical entanglements formed by more concentrated polymer solutions (low initial water content in the hydrogel). ${ }^{16}$

According to Table I, the porosity values ranged from 75 up to $90 \%$, which suggests that dexT70-VA hydrogels are formed by an interconnected structure. Interestingly, the porosity values achieved in this work are higher than other values described for polymeric matrices with the same pore range. ${ }^{6}$ This is an interesting characteristic of these hydro- 

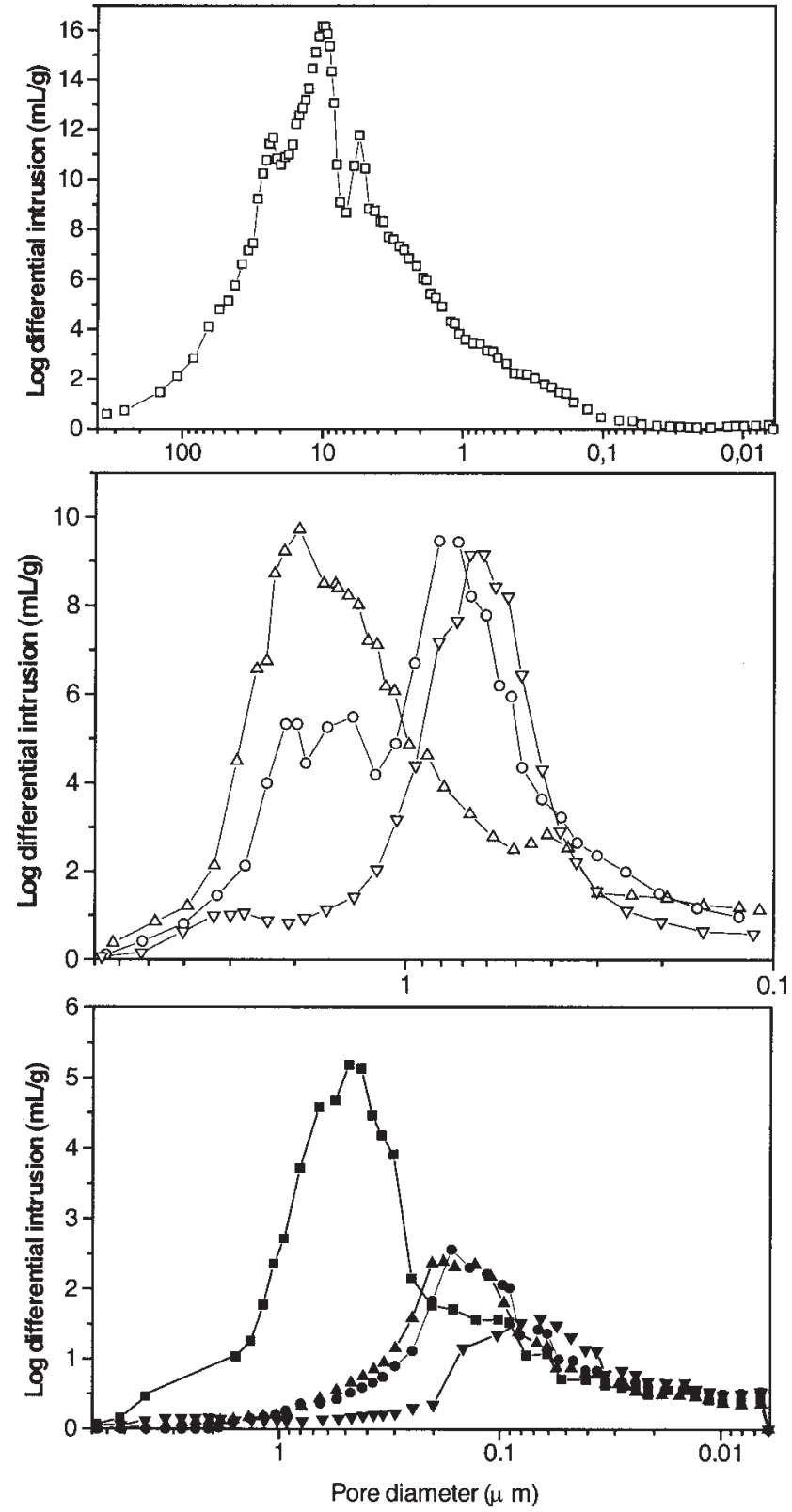

Figure 3. Pore size distribution (log differential intrusion against diameter) of dexT70-VA hydrogels with DS 7.2\% $(\square, \square), 12.1 \%(\triangle, \mathbf{\Delta})$, $22.4 \%(\bigcirc, \bullet)$, and $31.5 \%(\nabla, \nabla)$ and initial water content of $92 \%$ (open symbols) and $80 \%$ (solid symbols).

gels, which may have particular significance for their potential application in tissue engineering.

\section{Structural Analysis by NA}

Despite this technique being mostly used to measure surface areas in powders and porous networks, it can also provide useful information about pore size in the mesoporous range $(<0.050 \mu \mathrm{m})$. From the isotherms illustrated in Figure 4, obtained for all hydrogels, it can be seen that they are all identical, with shapes similar to those of type IV and hystereses of type I, denoting the presence of mesopores. ${ }^{27}$ BET

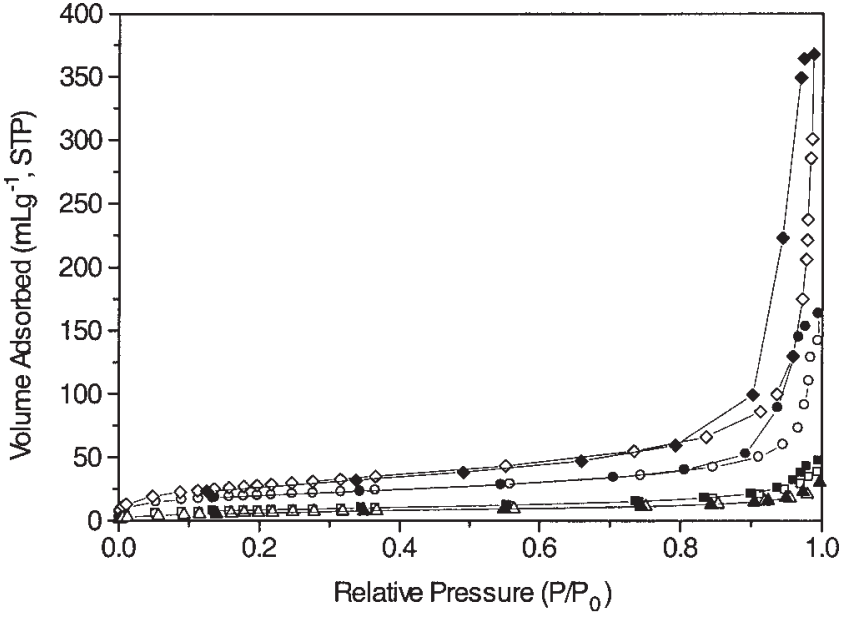

Figure 4. Nitrogen adsorption-desorption isotherms for dexT70-VA hydrogels: $92 \%$ DS $31.5 \%(\triangle, \mathbf{\Delta}), 80 \%$ DS $7.2 \%(\square, \square), 80 \%$ DS $12.1 \%(\bigcirc, \bullet)$, and $80 \%$ DS $31.5 \%(\nabla, \nabla)$. Open and closed symbols correspond to adsorption and desorption branches, respectively.

surface areas (Table II) were calculated from the corresponding isotherms with correlations higher than 0.99993 and C values ranging from 47 to 220 , demonstrating the validity of this method. ${ }^{27}$ Table II also shows that specific surface area increases as the initial water content decreases (at constant DS) and as the DS increases (for a constant initial water content) as a result of the presence of smaller pores (Table I).

The calculation of pore size distribution in dexT70-VA hydrogels was based on the BJH method (assuming cylindrical pores) using the desorption isotherms (Fig. 5), as recommended for comparisons with mercury intrusion. ${ }^{28,29}$ The modes of the distribution curves of the $80 \%$ dexT70-VA hydrogels (Table II and Fig. 5) show that pore sizes decrease as DS increases in agreement with the tendency found for the results of MIP. However, it should be emphasized that the absence of the plateau at high pressures in the isotherms of Figure 4 indicates that pores with larger diameters may exist, and therefore, these results should regarded with caution and complemented by MIP analysis. In fact, mercury porosimetry results (Fig. 3) show the occurrence of mercury intrusion either in macroporous or mesoporous regions, especially for

TABLE II. Characteristics of Swollen dexT70-VA Hydrogels with Different Initial Water Content and Degree of Substitution (DS), as Measured by Nitrogen Adsorption

\begin{tabular}{lcc}
\hline $\begin{array}{c}\text { DexT70-VA } \\
\text { Hydrogel }\end{array}$ & $\begin{array}{c}\text { Specific Surface } \\
\text { Area }\left(\mathrm{m}^{2} \mathrm{~g}^{-1}\right)^{\mathrm{a}}\end{array}$ & $\begin{array}{c}\text { Pore Size } \\
(\mathrm{nm})^{\mathrm{b}, \mathrm{c}}\end{array}$ \\
\hline $92 \%$, DS $31.5 \%$ & 23.8 & $--^{\mathrm{d}}$ \\
$80 \%$, DS $7.2 \%$ & 28.8 & 54 \\
$80 \%$, DS $12.1 \%$ & 69.0 & 44 \\
$80 \%$, DS $31.5 \%$ & 100.8 & 32 \\
\hline
\end{tabular}

\footnotetext{
${ }^{a}$ Determined by BET method.

${ }^{\mathrm{b}}$ Determined by BJH desorption branch.

${ }^{\mathrm{c}}$ Mode of the peaks.

${ }^{\mathrm{d}}$ No plateau was observed in the isotherms suggesting the existence of pore sizes above the detection limit of this technique.
} 

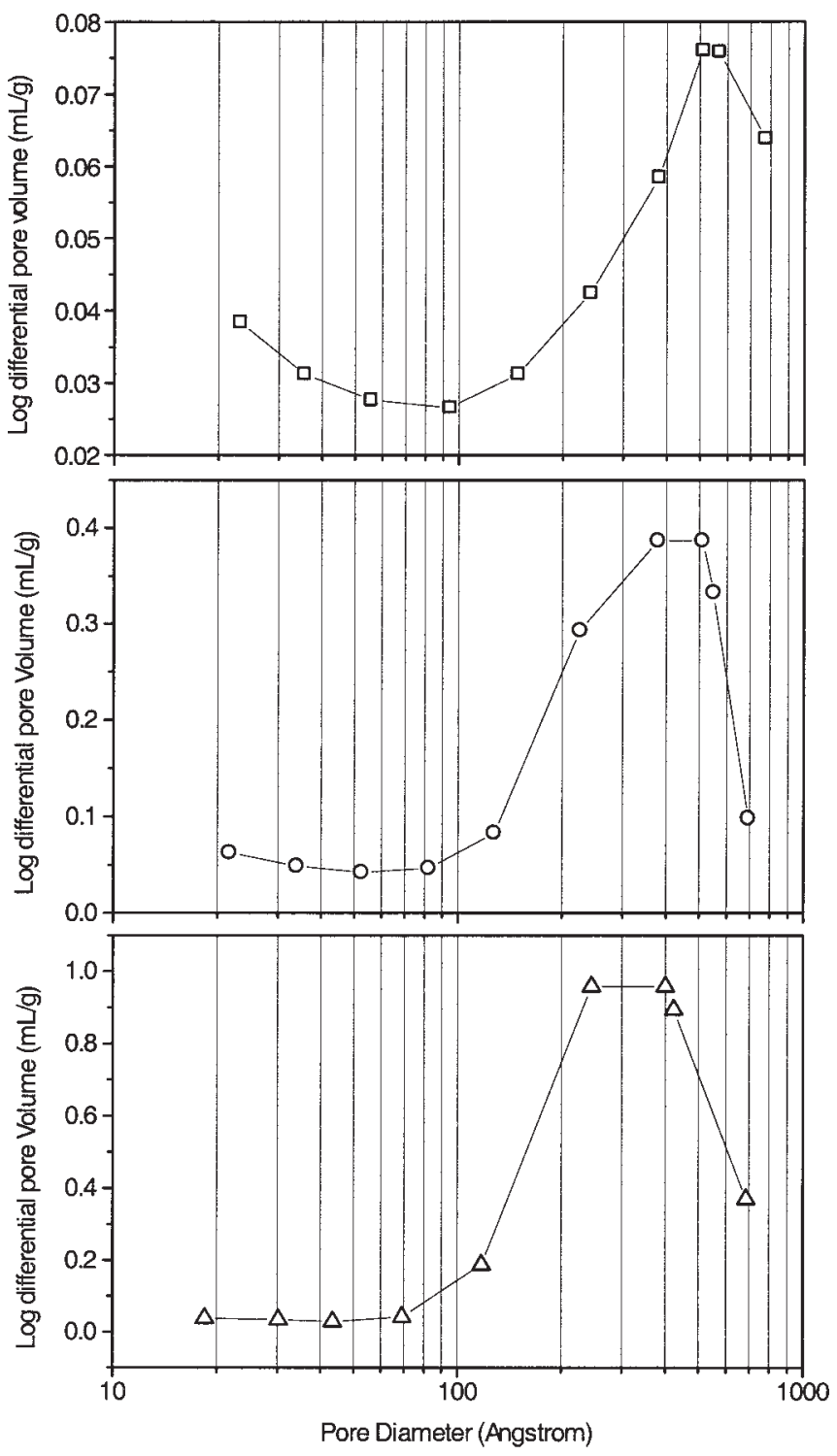

Figure 5. Pore size distribution $[d V / d \log (D)$, desorption branch] of dexT70-VA hydrogels with the same initial water content $(80 \%)$ and different DS as determined by nitrogen adsorption analysis: ( $\square$ ) DS 7.2\%; (O) DS 12.1\%; ( $\triangle$ ) DS 31.5\%.

hydrogels with low DS. Nevertheless, it should be noted that MIP is more reliable in the macroporous range whereas NA is recommended for meso- and micropore ranges. Moreover, the pore volumes calculated from gas adsorption are considerably smaller than those determined from mercury porosimetry, indicating that the number of these pores in the network is practically negligible.

\section{Structural Snalysis by SEM}

SEM provides information about pore geometry and size according to their specific location in the hydrogel, and therefore gives relevant information about the homogeneity/ heterogeneity of the hydrogel network. For this purpose, SEM pictures were taken at the surface and interior of the hydrogels at two different radial positions (Fig. 6). Figures 7 and 8 display SEM photographs taken at $\beta$ and $\delta$ locations (surface and interior inner regions of hydrogels), respectively.

Figures 7(A) and (B) show surface images from hydrogels with different initial water content (92 and 80\%, respectively) and the same DS (7.2\%). In both cases, hydrogels are covered by irregular pores with sizes ranging from $2-10 \mu \mathrm{m}$, in some places covered by a thin "skin" of polymer. Identical structure was also observed for hydrogel DS 12.1\% (data not shown). The presence of a polymeric "skin" has been described for other hydrogels, ${ }^{6,30}$ and is probably related to the collapse of surface pores by a freeze-drying process mainly in hydrogels with large pores and thin walls. A totally different structure was found either in 92 or $80 \%$ dexT70-VA DS $22.4 \%$ hydrogels [Fig. 7(C) and (D), respectively]. These networks are covered by pores with circular and elliptical geometries, ranging from 0.4 upon $8 \mu \mathrm{m}$ (Table III). In addition, the pores in hydrogel $80 \%$ DS $22.4 \%$ are larger than the ones observed in $92 \%$ DS $22.4 \%$ hydrogel. Identical structures were found in location $\alpha$ (outer radius, Fig. 6), indicating that the pictures of Figure 7 can be considered as representative of the overall surface.

Figure 8 shows a similar comparison for both hydrogels with respect to the interior location $\delta$ (inner radius, Fig. 6). Hydrogel 92\% DS 7.2\% exhibits an open 3D and homogeneous network structure, with large pores [Fig. 8(A) and Table III]. In contrast, hydrogels $80 \%$ DS 7.2\% [Fig. 8(B)] and $92 \%$ DS $22.4 \%$ [Fig. 8(C)] exhibit a lower average pore size (Table III) and a heterogeneous network structure, showing well-delimited regions with different pore sizes and geometries. Finally, in 92 and 80\% DS $12.1 \%$ (data not shown), and $80 \%$ DS $22.4 \%$ [Fig. 8(D)] hydrogels, their interior structures were more regular than the ones found in $80 \%$ DS $7.2 \%$ and $92 \%$ DS $22.4 \%$. Either small or large pores were mixed in the overall interior of hydrogel and delimited poreregions were not observed. Taken into account all hydrogels (Table III), in general, the average pore size (corresponding to the mode of the pore size distribution) decreased as the DS increased and the initial water content decreased. Regarding the outer part of hydrogel interiors ( $\chi$ in Fig. 6), our results indicate that the networks are more open at this location than at the inner part (considering the mode of pore size distributions) and either small or large pores are mixed in the overall region.

The results of Table III confirm that all dext70-VA hydrogels are clearly macroporous (with pore sizes far beyond 50 $\mathrm{nm}$ ), in accordance with the results obtained by mercury porosimetry. The discrepancies in the absolute values of pore

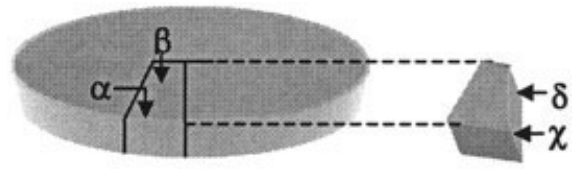

Figure 6. Location of the surface $(\alpha, \beta)$ and interior $(\chi, \delta)$ regions of the hydrogel evaluated by SEM. 

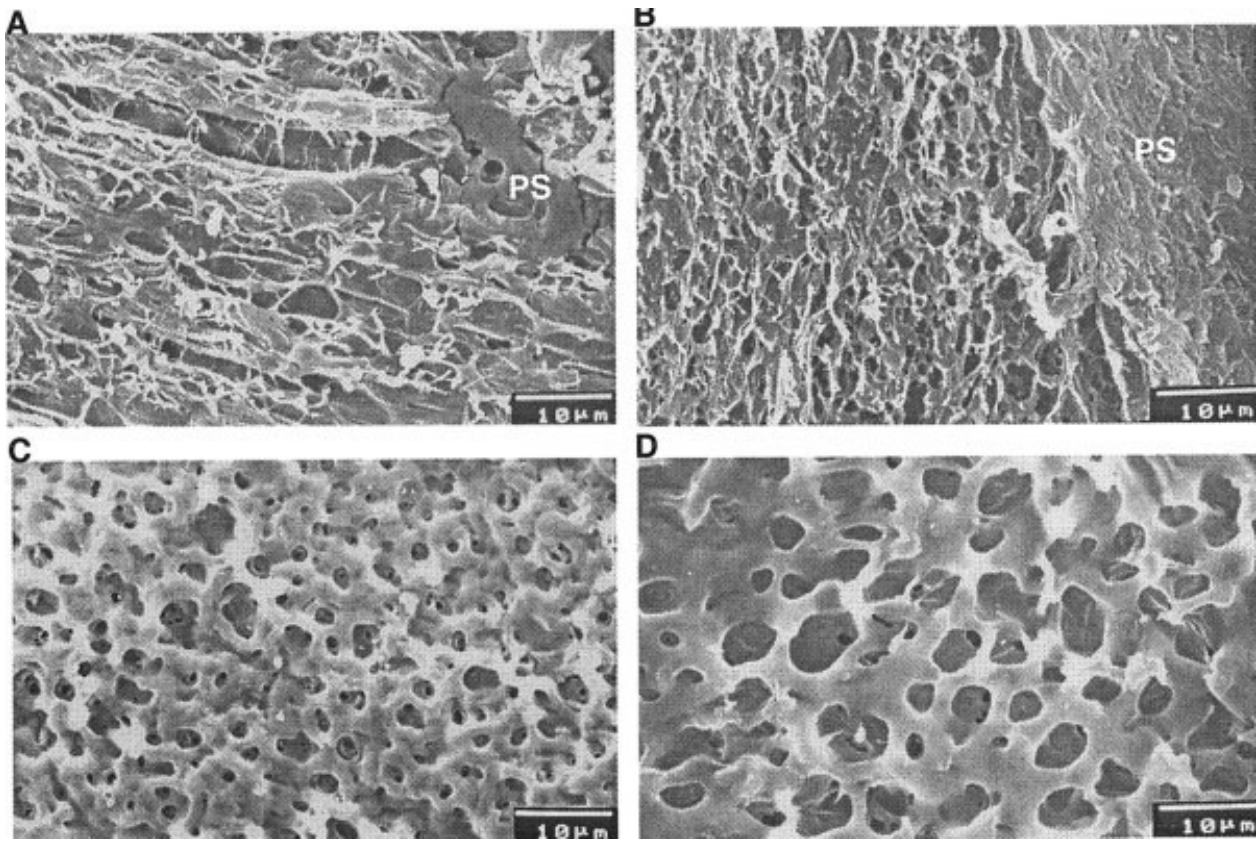

Figure 7. Scanning electron micrographs of surface region (inner radius, $\beta$ ) from swollen dexT70-VA hydrogels with different DS and initial water contents. (A) $92 \%$, DS $7.2 \%(\times 2000)$; (B) $80 \%$, DS $7.2 \%$ $(\times 2000)$; (C) $92 \%$, DS 22.4\% (×2000), and (D) $80 \%$, DS $22.4 \%(\times 2000)$. In $(A)$ and $(B)$ there is a polymeric "skin" (PS) covering the pores.

sizes estimated by both techniques are likely due to distinct measuring principles, size definitions, and sampled regions; however, similar trends were found for the effect of DS and initial water content in the hydrogel pore size. In addition, the results of this work show that dexT70-VA hydrogels are higher macroporous than other dextran-based hydrogels. For instance, Kim et al. ${ }^{12}$ reported that dextran-methacrylate hydrogels DS $9 \%$ and $24 \%$ had an average pore diameter in
A

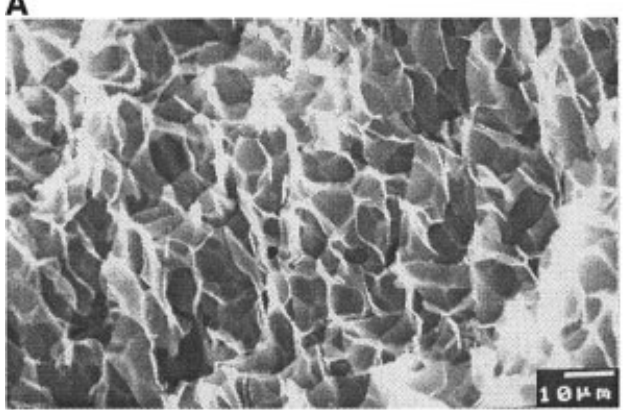

C

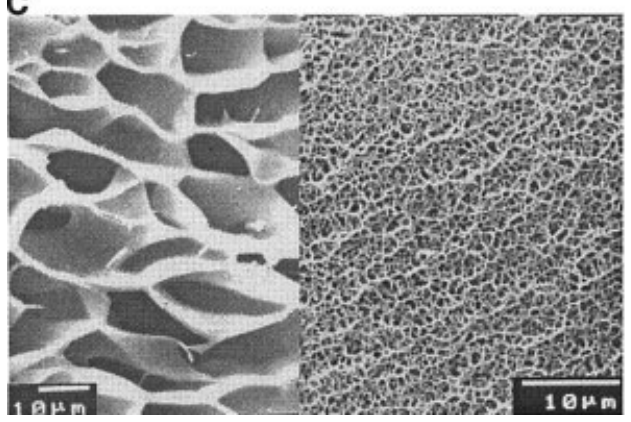

B

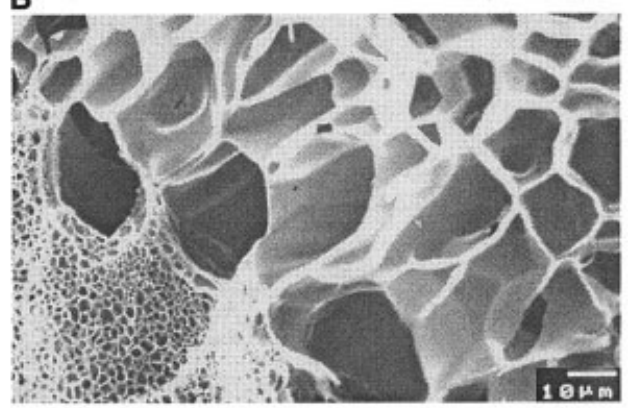

D

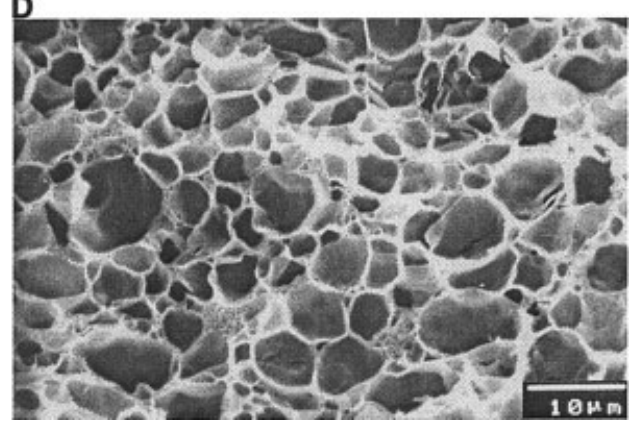

Figure 8. Scanning electron micrographs of interior region (inner radius, $\delta$ ) from swollen dexT70-VA hydrogels with different DS and initial water contents. (A) $92 \%$, DS $7.2 \%(\times 1000)$; (B) $80 \%$, DS $7.2 \%$ $(\times 1000)$; (C) $92 \%$, DS $22.4 \%(\times 2000$ and $\times 1000$, at two different locations in the sample), and (D) $80 \%$, DS $22.4 \%(\times 2000)$. 


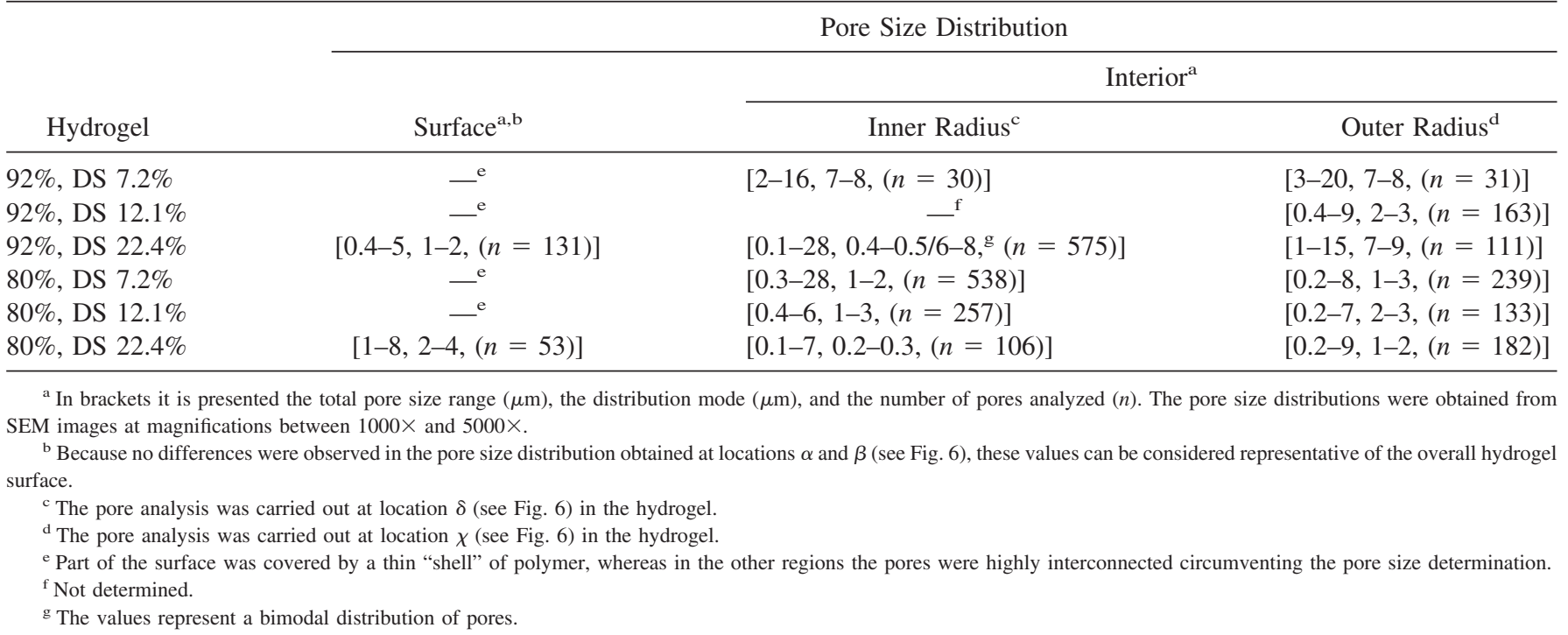

the mesoporous range $(2 \mathrm{~nm}<\mathrm{d}<50 \mathrm{~nm})$. The larger pores found in the present work for dexT70-VA hydrogels when compared to the photochemically generated dextran-methacrylate hydrogels may be related to the lower conversion of the vinyl groups into intermolecular linkages (intramolecular linkages do not contribute for the 3D structure and thus for pore formation). This lower conversion may be a consequence of the free-radical polymerization used in this work in contrast to the photo polymerization used in dextran-methacrylate hydrogels, the specific nature of the vinyl group used (acrylate/methacrylate), and/or to the regioselectivity achieved in the macromonomers (unfortunately, the regioselectivity pattern in dextran-methacrylate macromonomer was not reported).

\section{Structural Analysis by the Equilibrium Swelling Theory}

The structural properties of dexT70-VA hydrogels were also evaluated by a modeling approach previously used by us for nonsterilized networks. ${ }^{16}$ The average molecular weight between crosslinks $\left(\bar{M}_{c}\right)$ of dexT70-VA hydrogels was determined using the equilibrium swelling theory of Flory and Rehner, ${ }^{17}$ as previously described. ${ }^{16}$ This thermodynamic theory states that a crosslinked polymer gel, which is immersed in a fluid and allowed to reach equilibrium with its surrounding, is subject to two opposing forces, the thermodynamic force of mixing and the retractive force of the polymer chains. At equilibrium, these two forces are equal, which allows the calculation of $\bar{M}_{c}$ according to Equation (1). This theory is valid when the hydrogels are neutral, swelling is isotropic, and the crosslinks are tetrafunctional (four chains are connected at one crosslink point). After the calculation of $\bar{M}_{c}$ values, the average mesh size $(\xi)$ was calculated according to Equation (2) and (3). The mesh size is defined as the average linear distance between two adjacent crosslinks ${ }^{1}$ and characterizes the network and the effective space available for solute diffusion. ${ }^{31}$ It is very often used as a structural parameter in describing the size of the pores and thus can be regarded as the average pore size. ${ }^{1}$

According to the results of Table IV, the average pore sizes ranged from ca. 3 to $21 \mathrm{~nm}$ for the different hydrogels, and thus are highly underestimated compared to the values achieved by SEM, MIP, and NA. Despite the large differences in the average pore sizes, both approaches show that hydrogels with low initial water content and high DS values present lower average pore sizes than hydrogels with high initial water content and low DS values.

Discrepancies between average pore sizes calculated by the equilibrium swelling theory of Flory-Rehner and other approaches have been also reported elsewhere. Hennink et al. $^{20}$ have noticed that the average pore size of dextranmethacrylate hydrogels, calculated through the equilibrium swelling theory, was underestimated when compared to the values obtained by protein release experiments. Furthermore,

TABLE IV. Network Properties ${ }^{\mathrm{a}}$ of dexT70-VA Gels as a Function of the Initial Water Content and DS

\begin{tabular}{lcccrr}
\hline $\begin{array}{c}\text { DexT70-VA } \\
\text { Hydrogel }\end{array}$ & SRE $^{\mathrm{b}}$ & $v_{2, r}{ }^{\mathrm{c}}$ & $v_{2, s}{ }^{\mathrm{d}}$ & $\begin{array}{c}\bar{M}_{c}{ }^{\mathrm{e}} \\
(\mathrm{g} / \mathrm{mol})\end{array}$ & $\begin{array}{r}\xi^{\mathrm{f}} \\
(\mathrm{nm})\end{array}$ \\
\hline $92 \%$, DS 7.2\% & 36.6 & 0.104 & 0.0277 & 14695.2 & 20.5 \\
$92 \%$, DS 12.1\% & 14.4 & 0.123 & 0.0706 & 5686.3 & 9.3 \\
$92 \%$, DS 22.4\% & 11.5 & 0.133 & 0.0829 & 4496.7 & 7.9 \\
$80 \%$, DS 7.2\% & 10.2 & 0.184 & 0.0984 & 4102.5 & 7.1 \\
$80 \%$, DS 12.1\% & 5.86 & 0.228 & 0.167 & 1596.3 & 3.7 \\
80\%, DS 22.4\% & 5.32 & 0.207 & 0.164 & 1549.2 & 3.7 \\
80\%, DS 31.5\% & 4.80 & 0.219 & 0.195 & 1115.7 & 2.9 \\
\hline
\end{tabular}

${ }^{\text {a }}$ Average of two independent measurements.

${ }^{\mathrm{b}}$ Swelling ratio at equilibrium.

${ }^{\mathrm{c}}$ Polymer fraction of the gel after gel formation.

${ }^{\mathrm{d}}$ Polymer fraction at equilibrium swelling.

e Average molecular weight between the crosslinks.

${ }^{\mathrm{f}}$ Average mesh size. 
an underestimation of the hydrogel mesh size using FloryRehner analysis was also reported by Reinhart and Peppas for poly(vinyl alcohol) hydrogels crosslinked with glutaraldehyde. $^{32}$

Typical values of average pore size calculated through the Flory-Rehner analysis for hydrogels were in the micropore and mesopore ranges (5.5-11.9 nm for poly(ethylene glycol) hydrogels, ${ }^{21} 3.7-32.1 \mathrm{~nm}$ for poly(vinyl alcohol) hydrogels, ${ }^{19}$ $1.6-2.4 \mathrm{~nm}$ for poly(hydroxyethyl methacrylate) hydrogels ${ }^{19}$ and 4-11 nm for dextran-methacrylate hydrogels ${ }^{20}$ ). To our knowledge, this modeling approach has not been used on macroporous hydrogels such as the ones described in this work, and thus may not describe adequately the network properties. In fact, as the average pore size of dexT70-VA hydrogels increases according to MIP analyses, higher differences were found between those values and the ones obtained through the Flory-Rehner analysis. The underestimation of the average pore size values may result from an underestimation of $\bar{M}_{c}$ values. Due to several vinyl groups attached to each dextran chain, junctions with higher functionalities than tetrafunctional assumed by the Flory-Rehner theory can be expected for dexT70-VA hydrogels. This will decrease the $\bar{M}_{c}$ values and thus the average pore size.

Another possible explanation for the differences found in the average pore sizes obtained by MIP and Flory-Rehner analysis may result from differences in the sample state. For Flory-Rehner analysis, swollen hydrogels were used while in SEM; MIP and NA the samples were first cryofixed and then lyophilized. Although it has been reported that cryofixation and freeze-drying processes have a minimal disturbance in the network structure, ${ }^{12}$ we further investigated this issue. For that purpose, swollen $80 \%$ dexT70-VA $12.1 \%$ hydrogels were characterized by an alternative methodology to the equilibrium swelling theory. In this case, rheological tests were performed to obtain the shear modulus, $G^{\prime}$, which were converted into $\bar{M}_{c}$ values by the phantom network theory ${ }^{33}$ according to Equation (6):

$$
G^{\prime}=\frac{\rho R T}{\bar{M}_{c} \nu_{2, s}{ }^{\frac{1}{3}}}\left(1-\frac{2 \bar{M}_{c}}{M_{n}}\right)
$$

where $\rho$ is the polymer density, $R$ is the gas constant ( 8.314 $\left.\mathrm{JK}^{-1} \mathrm{~mol}^{-1}\right), T$ is the temperature $(298 \mathrm{~K}), \bar{M}_{n}$ is the number average molecular weight of dextran $(39,940 \mathrm{Da})$ and $\nu_{2, s}$ is the polymer fraction at equilibrium swelling (Table IV). It should be noted that the phantom network theory allows for movement of the crosslink junctions through rearrangements of the chains and also account for the presence of terminal chains in the network structure. ${ }^{33}$

The $G^{\prime}$ value for the swollen $80 \%$ dexT70-VA $12.1 \%$ hydrogel was $11,123 \pm 2353 \mathrm{~Pa}(n=3)$, which yields a $\bar{M}_{c}$ value of 3,944,517 $\pm 744,076 \mathrm{gmol}^{-1}$ after using Equation (6). The conversion of $\bar{M}_{c}$ value through Equations (2) and (3) yields an average pore size of $183 \mathrm{~nm}$. This value is quite close to that value found by MIP for the corresponding hydrogel $(200 \mathrm{~nm})$, and suggests that the sample preparation does not explain the differences found in the average pore sizes as determined by MIP, SEM (and even NA), and the Flory-Rehner model.

\section{CONCLUSIONS}

Three analytical techniques (MIP, NA, and SEM) were used to analyze pore structure of swollen dextran-acrylate hydrogels generated chemoenzymatically. The results of MIP have shown that the overall networks were essentially macroporous (with pores up to several micrometers); however, gas adsorption also revealed a small amount of mesopores. SEM analysis confirmed these findings and showed that the interior morphology varied according to the DS and the initial water content of the hydrogels. A polymeric "skin" covering the pores surface of hydrogels (specially those with lower DS) was sometimes observed. The comparison of the SEM and MIP results with the ones obtained by the Flory-Rehner model shows that this last approach was inadequate and highly underestimated the average pore size of dexT70-VA hydrogels, mainly for networks with large pore sizes. The results also suggest that the underestimation of the average pore size by the Flory-Rehner model is not caused by the sample state.

Finally, the results described in this work show that it is feasible to control the average pore size of dexT70-VA hydrogels by changing the DS of the dext70-VA macromonomer and/or the initial water content used in the hydrogel preparation. In fact, all the techniques revealed that pore size decreases as DS increases and/or the initial water content decreases. This is of crucial importance for the ultimate application of these networks as controlled release systems or as scaffolds for tissue engineering. The DS in dexT70-VA hydrogel affects the extent of crosslinking, which in turn, affects the pore size distribution of the resulting swollen network; on the other hand, the initial water content of the hydrogel (inversely related to the initial concentration of polymer chains) affect the extent of intermolecular/intramolecular linkages that will be formed, and thus the ultimate pore size distribution.

The authors thank Vitor Redondo for skilled technical assistance.

\section{REFERENCES}

1. Peppas NA, Bures P, Leobandung W, Ichikawa H. Hydrogels in pharmaceutical formulations. Eur J Pharm Biopharm 2000;50: 27-46.

2. Park K, Park H. Biocompatibility issues of implantable drug delivery systems. Pharm Res 1996;13:1770-1776.

3. Lee KY, Mooney DJ. Hydrogels for tissue engineering. Chem Rev 2001;101:1869-1879.

4. Nguyen KT, West JL. Photopolymerizable hydrogels for tissue engineering applications. Biomaterials 2002;23:4307-4314.

5. Meyvis T, De Smedt S, Stubbe B, Hennink W, Demeester J. On the release of proteins degrading dextran methacrylate hydrogels and the correlation with the rheologic properties of the hydrogels. Pharm Res 2001;18:1593-1599. 
6. Dziubla TD, Torjman MC, Joseph JI, Murphy-Tatum M, Lowman AM. Evaluation of porous networks of poly(2-hydroxyethyl methacrylate) as interfacial drug delivery devices. Biomaterials 2001;22:2893-2899.

7. Jen AC, Wake MC, Mikos AG. Review: Hydrogels for cell immobilization. Biotechnol Bioeng 1996;50:357-364.

8. Silver FH. Wound dressings and skin replacement. In: Silver $\mathrm{FH}$, editor. Biomaterials, medical devices and tissue engineering. London: Chapman \& Hall; 1994. p. 47-91.

9. Iza M, Woerly S, Danumah C, Kaliaguine S, Bousmina M. Determination of pore size distribution for mesoporous materials and polymeric gels by means of DSC measurements: Thermoporometry. Polymer 2000;41:5885-5893.

10. Everett DH. IUPAC manual of symbols and terminology for physicochemical quantities and units. Pure Appl Chem 1972; 31:579-638.

11. Behravesh E, Jo S, Zygourakis K, Mikos AG. Synthesis of in situ cross-linkable macroporous biodegradable poly(propylene fumarate-co-ethylene glycol) hydrogels. Biomacromolecules 2002;3:374-381.

12. Kim S-H, Chu C-C. Pore structure analysis of swollen dextranmethacrylate hydrogels by SEM and mercury intrusion porosimetry. J Biomed Mater Res: Appl Biomater 2000;53:258-266.

13. Kang H-W, Tabata Y, Ikada Y. Fabrication of porous gelatin scaffolds for tissue engineering. Biomaterials 1999;20:1339-1344.

14. Woerly S, Marchand R, Lavallée C. Interactions of copolymeric poly(glyceryl methacrylate)-collagen hydrogels with neural tissue: Effects of structure and polar groups. Biomaterials 1991; 12:197-203.

15. Woerly S, Pinet E, De Robertis L, Bousmina M, Laroche G, Roitback T, Vargová L, Syková E. Heterogeneous PHPMA hydrogel for tissue repair and axonal regeneration in the injured spinal cord. In: Schoichet MS, Hubbell JA, editors. Polymers for tissue engineering. Utrecht: VSP; 1998. p. 343-373.

16. Ferreira L, Gil MH, Dordick JS. Enzymatic synthesis of dextran-containing hydrogels. Biomaterials 2002;23:39573967.

17. Flory PJ, Rehner R. Statistical mechanics of cross-linked polymer networks. II. Swelling. J Chem Phys 1943;11:521526.

18. Peppas NA, Moynihan HJ, Lucht LM. The structure of highly crosslinked poly(2-hydroxyethylmethacrylate) hydrogels. J Biomed Mater Res 1985;19:397-411.
19. Canal T, Peppas NA. Correlation between mesh size and equilibrium degree of swelling of polymeric networks. J Biomed Mater Res 1989;23:1183-1193.

20. Hennink WE, Talsma H, Borchert JCH, De Smedt SC, Demmester J. Controlled release of proteins from dextran hydrogels. J Control Release 1996;39:47-55.

21. Stringer JL, Peppas NA. Diffusion of small molecular weight drugs in radiation-crosslinked poly(ethylene oxide) hydrogels. J Control Release 1996;42:195-202.

22. Washburn EW. Note on a method of determining the distribution of pore sizes in a porous material. Proc Natl Acad Sci USA 1921;7:115-116.

23. Brunauer S, Emmett PH, Teller E. Adsorption of gases in multimolecular layers. J Am Chem Soc 1938;60:309-319.

24. Barrett EP, Joyner LG, Hallendo PB. The determination of pore volume and area distributions in porous substances-I. Computations from nitrogen isotherms. J Am Chem Soc 1951;73: 373-380.

25. Meyvis TKL, De Smedt SC, Demeester J, Hennink WE. Rheological monitoring of long-term degrading polymer hydrogels. J Rheol 1999;43:933-950.

26. Ferreira L, Rafael A, Lamghari M, Barbosa MA, Gil MH, Cabrita AMS, Dordick JS. Biocompatibility of chemoenzymatically derived dextran-acrylate hydrogels. J Biomed Mater Res 2004;68:584-596.

27. Gregg SJ, Sing KSW. Adsorption, surface area and porosimetry. 2nd ed. London: Academic Press Inc.; 1991.

28. Van Brackel J, Modry S, Svatá M. Mercury porosimetry: State of the art. Powder Technol 1981;29:1-12.

29. Moscou L, Lub S. Practical use of mercury porosimetry in the study of porous solids. Powder Technol 1981;29:45-52.

30. Chirila TV, Constable IJ, Crawford GJ, Vijayasekaran S, Thompson DE, Chen Y-C, Fletcher WA, Griffin BJ. Poly(2hydroxyethyl methacrylate) sponges as implant materials: In vivo and in vitro evaluation of cellular invasion. Biomaterials 1993;14:26-38.

31. Lustig SR, Peppas NA. Solute diffusion in swollen membranes. IX. Scaling laws for solute diffusion in gels. J Appl Polym Sci 1988;36:735-747.

32. Reinhart CT, Peppas NA. Solute diffusion in swollen membranes. Part II. Influence of crosslinking on diffusive properties. J Membr Sci 1984;18:227-239.

33. Bell CL, Peppas NA. Biomedical membranes from hydrogels and interpolymer complexes. Adv Polym Sci 1995;122:125-175. 